\title{
Disease, Disorder or Finding
}

National Cancer Institute

\section{Source}

National Cancer Institute. Disease, Disorder or Finding. NCI Thesaurus. Code C7057.

A condition that is relevant to human neoplasms and non-neoplastic disorders. This includes observations, test results, history and other concepts relevant to the characterization of human pathologic conditions. 